\title{
Delayed presentation of port-site metastasis from an unknown gastrointestinal malignancy following laparoscopic cholecystectomy
}

\author{
Siddharth Rao ${ }^{1}$, mBвs, Ms, Anil Rathod ${ }^{1}$, mBBs, Ashok Kamble ${ }^{1}$, mBвs, ms, Dilip Gupta ${ }^{1}$, MBвs, MS
}

\begin{abstract}
Port-site metastasis (PSM) is often encountered during laparoscopic surgery in patients with malignancy. We report a 45-year-old woman who presented with a single PSM from papillary adenocarcinoma after undergoing laparoscopic cholecystectomy for calculus cholecystitis. Post cholecystectomy, a diagnosis of chronic cholecystitis was confirmed on histopathology. The patient presented with a mass at the site of epigastric port 28 months after surgery. PSM was suspected on clinical examination, which was supported by findings on computed tomography and further confirmed by fine-needle aspiration cytology of the lump. The patient underwent surgical clearance of the mass, and histopathological examination proved the lesion to be papillary adenocarcinoma. The site of the primary tumour was not detected even after thorough examination. Based on the histopathology report following local surgical clearance, the patient was started on chemotherapy. This case is unusualbecause of the long delay prior to the presentation of PSM and the unknown primary malignancy.
\end{abstract}

Keywords: gallbladder surgery, laparoscopic cholecystectomy, laparoscopic surgery adverse effects, port-site metastasis

\section{INTRODUCTION}

The advent of laparoscopic oncologic surgery has brought with it the enigmatic problem of port-site metastasis (PSM). PSM is extensively reported in the literature and has been encountered in almost all areas of laparoscopic oncologic surgery, videoassisted thoracoscopic surgery and robot-assisted oncologic surgery. ${ }^{(1)}$ However, all reports of PSM have occurred following elective oncologic surgical procedures that were performed for the purposes of either staging or complete resection of tumours. PSM has also been reported after laparoscopic cholecystectomy (LC) in patients with unsuspected gallbladder malignancy that was proven as malignancy only on histopathology. ${ }^{(2)}$

We report the case of a 45 -year-old woman with a single PSM from papillary adenocarcinoma, who presented 28 months after she had undergone LC for calculus cholecystitis. The primary malignancy remained elusive in our patient even after thorough investigation - there was no evidence of malignancy anywhere in the body, except for a peripancreatic lymph node that had no indication of malignancy. This gave rise to suspicion of hidden or occult malignancy, probably in the gallbladder or pancreas, and also created confusion regarding the institution of postoperative chemotherapy and the selection of appropriate chemotherapeutic agents for our patient.

To the best of our knowledge, only two patients with PSM without evidence of primary malignancy have been previously reported in the literature. In both these cases, the metastases occurred within 11 months of LC. ${ }^{(3)}$ Our patient, who is only the third report of PSM without evidence of primary malignancy in the literature, was unique due to the long delay of 28 months prior to the presentation of PSM following LC.

\section{CASE REPORT}

A 45-year-old woman who presented to the Department of Surgery, Mahatma Gandhi Institute of Medical Sciences (MGIMS), Sevagram, India, with symptoms of dyspepsia was diagnosed with cholelithiasis. She was admitted to the hospital on October 11, 2007, and underwent LC on October 15, 2007, after full investigations and preanaesthetic checkups. Histopathological analysis of the excised specimen confirmed the diagnosis of calculus cholecystitis. The patient had an uneventful recovery and her symptoms of dyspepsia disappeared. She was discharged on October 20, 2007, without any wound complication such as infection or gaping.

However, 28 months after LC, the patient noticed a painless lump in the epigastric region which was insidious in onset and slowly progressive. She was readmitted on March 29, 2010, and clinical examination revealed a hard fixed mass $43 \mathrm{~mm}$ at the site of epigastric port of the LC. All other port sites were normal. Computed tomography (CT) of the abdomen showed an ill-defined, hypodense mass, measuring $42 \mathrm{~mm} \times 46 \mathrm{~mm}$ in size, with enhancing areas and necrosis in anterior abdominal wall in the right upper para median region that could have been metastatic in origin (Fig. 1). There was also an enlarged peripancreatic lymph node $34 \mathrm{~mm} \times 26 \mathrm{~mm}$ in size. There was no evidence of any primary malignancy on CT of the abdomen. Fine-needle aspiration cytology from the epigastric mass, performed on March 27, 2010, was suggestive of mucinsecreting adenocarcinoma. A further search for the primary malignancy could not be undertaken due to economic constraints.

The patient underwent wide surgical clearance of the mass encompassing a 2-cm margin of normal tissue on April 12, 2010 


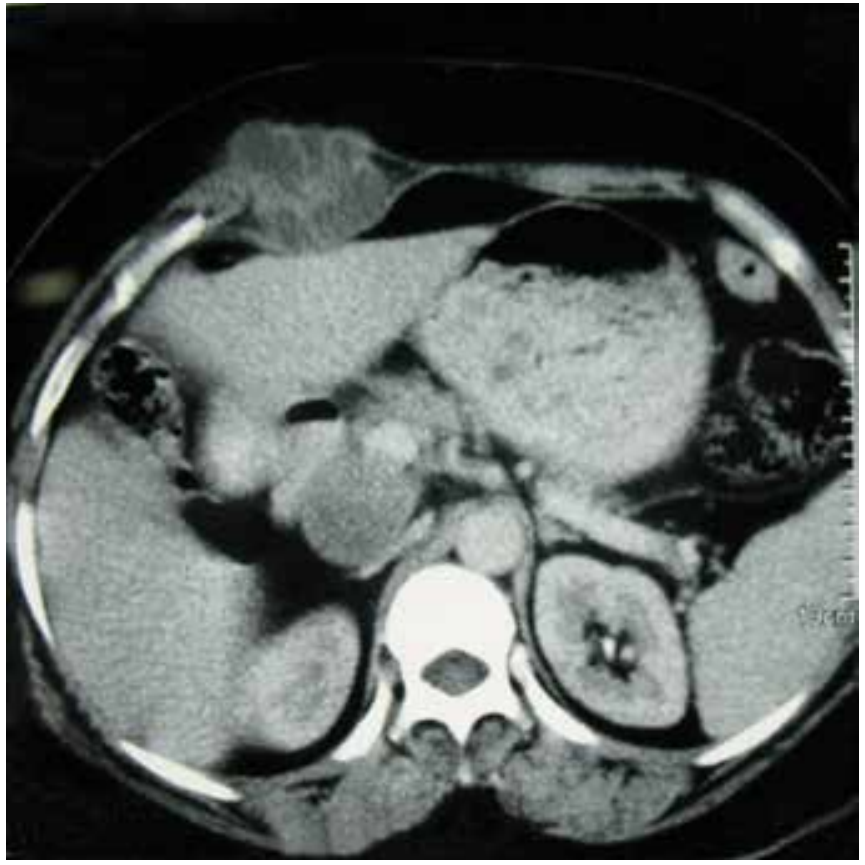

Fig. 1 Axial contrast-enhanced CT image of the abdomen shows port-site metastasis as an ill-defined, hypodense mass.

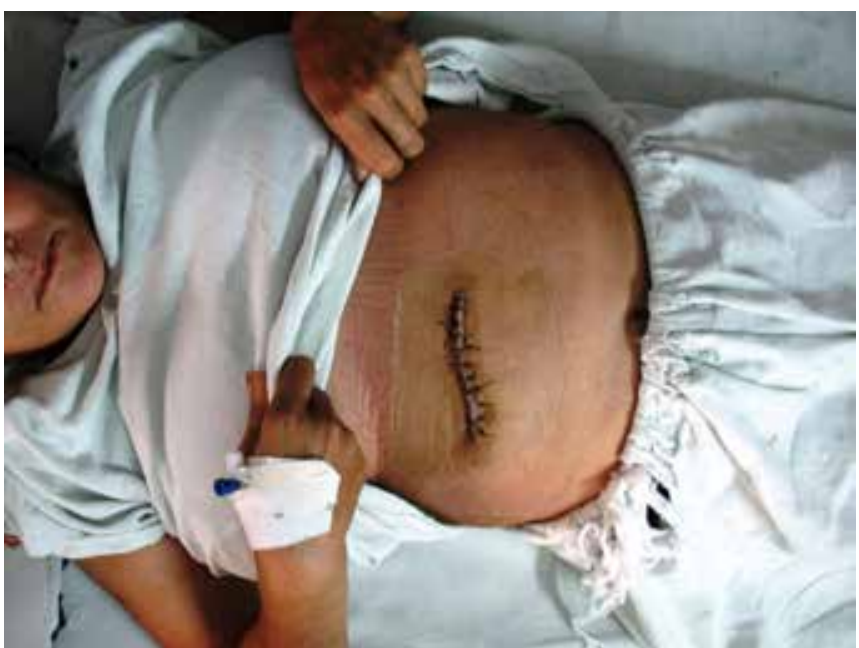

Fig. 2 Photograph shows the site of the surgical excision of the port-site metastasis.

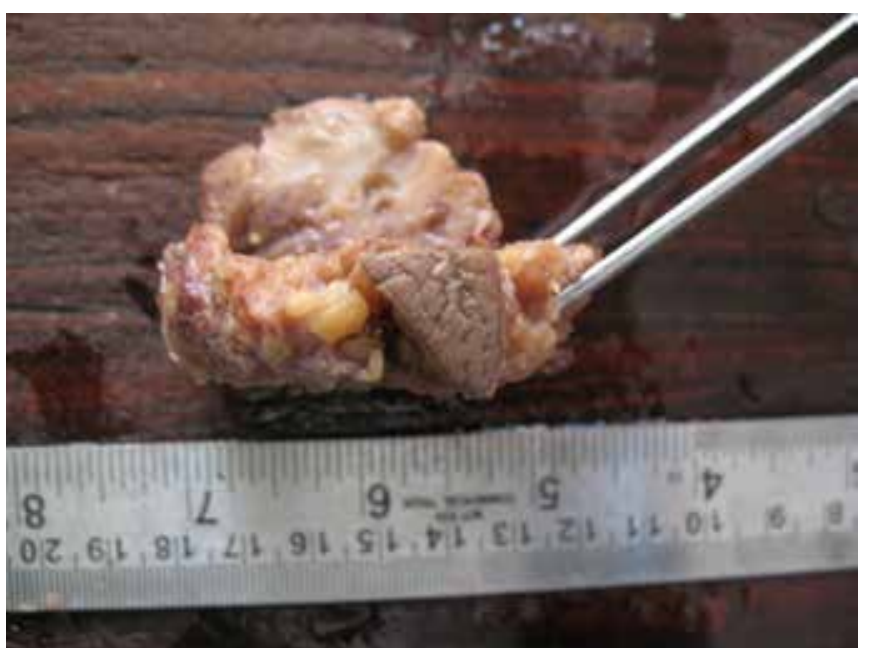

Fig. 3 Photograph of the surgical specimen shows skin and part of the malignant mass.
(Figs. 2 \& 3). The tumour was found to extend from the peritoneum to the skin. Histopathological analysis of the excised specimen was suggestive of papillary adenocarcinoma. The patient underwent upper gastrointestinal endoscopy and colonoscopy, which showed no evidence of any pathology. CT-guided fine-needle aspiration cytology of the peripancreatic lymph node, although attempted, was inconclusive. In view of the histopathological findings, the patient was started on a course of chemotherapy. She remains on regular follow-up. However, no evidence of any metastatic deposit has been noted at any local or distal predicted site to date.

\section{DISCUSSION}

The mechanism of metastases and the various factors favouring development of PSM have been discussed by various authors, ${ }^{(4,5)}$ with many studies also suggesting the possible reasons for finding PSM in patients undergoing oncologic surgeries. ${ }^{(4,5)}$ Laparoscopic surgeries are associated with several desirable advantages such as better tumour staging, lesser pain, quicker recovery and shorter hospitalisation, rendering them a widely adopted option for patients with intra-abdominal malignancies. ${ }^{(6)}$ However, the occurrence of PSM in patients following laparoscopic procedures is a shortcoming that threatens to negate all its advantages. The exact incidence of PSM is difficult to ascertain, as it requires the exact number of not only the laparoscopic surgeries performed but also PSM developed in any given population. (1) That being the case, the reported incidence of PSM in the literature varies from $0.71 \%{ }^{(7)}$ to $21 \%{ }^{(8)}$ PSM is even known to occur in patients undergoing staging or diagnostic laparoscopy where no tumour has been handled.(9)

Although the exact mechanism of metastasis is unknown, it appears to be multifactorial in nature. One unlikely factor that has been suggested is haematogenous spread. ${ }^{(4)}$ Direct implantation of the tumour cells is likely to be another major contributory factor of PSM, as tumour contamination on operating instruments has been reported. ${ }^{(9)}$ Such cells might, in turn, contaminate the trocar site, leading to the subsequent development of PSM. ${ }^{(9)}$ Although PSM can occur at any of the port sites, the operating port is more commonly involved. ${ }^{(10)}$ This may be attributed to the fact that the operating port encounters higher contamination by tumour cells when compared to the assistant's ports. Injury to the peritoneum and abdominal wall at the trocar sites, with the presence of blood clots and injured tissue, have also been shown to increase the likelihood of tumour implantation due to the presence of fibrin that may trap the tumour cells.

As laparoscopy is usually performed in the presence of pneumoperitoneum, the type of gas used, the pressure and duration of surgery might influence tumour seeding. Although an intact peritoneum is resistant to the implantation of tumour cells, an injury, as produced by the introduction of a trocar, increases the chances of tumor seeding. Also, carbon dioxide has been shown to irritate the peritoneum, causing inflammatory changes and acidosis, along with impaired macrophage 
function, which favours the implantation of tumour cells. ${ }^{(8)}$ According to $\mathrm{Wu}$ et al, carbon dioxide pneumoperitoneum at $10 \mathrm{mmHg}$ increases wound implantation in cases of intraoperative tumour spillage. ${ }^{(5)}$ Such difficulties can be addressed to a certain extent by using inert gases such as helium for creating the pneumoperitoneum or by performing a gasless laparoscopy. ${ }^{(9)}$

Surgical technique also determines the incidence of PSM following laparoscopic oncosurgery, which is associated with the surgeon's learning curve. Tumour handling and manipulation increases the chances of metastasis in both open and minimally invasive surgeries. ${ }^{(11)}$ The incidence of PSM following minimally invasive surgery has been found to decrease with increased experience of the surgeon. ${ }^{(12)}$ These findings re-emphasise that, to reduce the chances of tumour spillage, the basic oncologic surgery principles of avoiding manipulation and handling of the tumour should be followed in laparoscopic surgery as well.

The aerosolisation of tumour cells and subsequent contamination of the port sites have been reported. ${ }^{(13)}$ However, an extremely high volume of cells is required for metastases to occur by this route. ${ }^{(14)}$ The chimney effect, due to the presence of leaks around the trocar, has also been postulated as a cause of PSM, ${ }^{(14)}$ although this remains unclear. A possible reason might be the aerosolisation of the free floating malignant cells in the peritoneum, or due to fluid.

Several techniques have been proposed to decrease the chances of PSM, ${ }^{(15)}$ although with variable results. Some aim to decrease the inoculation of malignant cells by physical methods such as using an endobag while delivering the specimen, excision of the port site, use of helium insuffulation or gasless laparoscopy, and avoiding manipulation of the tumour. Povidone iodine when injected intraperitoneally has been shown to have tumoricidal activity. ${ }^{(16)}$ The use of topical intraperitoneal cytotoxic drugs has also been recommended. ${ }^{(16)}$ However, a study done by Tai et al that used topical oxaliplatin application in a rat injected with viable rat colon carcinoma cells found no statistically significant differences PSM rates of the treated and untreated layers of the abdominal wall. ${ }^{(17)}$

In view of the current literature, the best approach to limit the development of PSM following laparoscopic procedures would be to avoid direct tumour handling and strictly adhere to the principles of laparoscopic oncological surgery. However, the usefulness of such approaches is limited to oncologic surgeries where there is evidence or suspicion of malignancy. It might not be of significance in cases where there is no evidence of malignancy or the procedure is being performed for other reasons such as laparoscopic cholecystectomy. In such a scenario where patients develop PSM following laparoscopic procedures, in spite of all precautions being taken, the most probable reason for developing metastases might be the settling down of hitherto unknown circulating malignant cells at the injured site rather than a direct contamination by the tumour. The long delay between LC and PSM presentation in our patient suggests that this might have been the likely cause of PSM. The malignancy in our patient was also shown to be low grade, although evidence of any primary malignancy elsewhere in the body was not forthcoming.

This was the first case of PSM encountered at our centre in over 210 patients who have undergone LC at MGIMS Sevagram. Interestingly, PSM occurred without any obvious malignancy in our patient and might have been a metastatic manifestation of an occult primary. Few authors have reported PSM in patients with an unknown primary malignancy. ${ }^{(3)}$ In the two patients with such PSM reported in the literature, recurrences were noted 11 and 6 months after LC, respectively.(3) In contrast, PSM was only noted 28 months after LC in our patient, indicating a unique presentation with an unusually long delay.

The only finding in our patient was a single peripancreatic lymph node that showed no conclusive evidence of any pathology on CT-guided aspiration. The primary malignancy remained elusive even after thorough investigation. As mentioned earlier, the most probable mechanism of metastasis could be the settling down of free circulating malignant cells from an unknown primary malignancy in the raw area created by the laparoscopic instruments via one of the many possible implantation routes, such as haematological, lymphatic or direct route.

Surgeons opting for laparoscopic procedures should remain aware of the possibility of PSM presenting after a latency period ranging from a few months to three to four years. While many factors may be responsible for the development of PSM following laparoscopic surgeries, the most common is intraoperative spillage. The best treatment option for these patients is still debatable. We suggest that patients undergoing laparoscopic surgeries be followed up for extended durations to enable early detection of PSMs in case they do develop.

\section{REFERENCES}

1. Hung GU, Hsu HK, Kao CH, Chen KY, Chiu JS. Asymptomatic port-site metastasis following video-assisted thoracoscopic surgery detected by FDG-PET/CT. Clin Nucl Med 2010; 35:552-3.

2. $\mathrm{Hu}$ JB, Sun XN, Xu J, He C. Port site and distant metastases of gallbladder cancer after laparoscopic cholecystectomy diagnosed by positron emission tomography. World J Gastroenterol 2008; 14:6428-31.

3. Polychronidis A, Tsaroucha AK, Perente S, et al. Port-site metastasis of extrahepatic bile duct carcinoma after laparoscopic cholecystectomy without evidence of a primary tumour. Acta Chir Belg 2008; 108:768-70.

4. Iwanaka T, Arya G, Ziegler MM. Mechanism and prevention of port-site tumor recurrence after laparoscopy in a murine model. J Pediatr Surg 1998; 33:457-61.

5. Wu JS, Brasfield EB, Guo LW, et al. Implantation of colon cancer at trocar sites is increased by low pressure pneumoperitonium. Surgery 1997; 122:1-7.

6. Toouli J, Cox MR. Minimal access surgery of the gastrointestinal tract. Aust N Z Surg 1995; 65:525-32.

7. Ziprin P, Ridgway PF, Peck DH, Darzi AW. The theories and realities of port-site metastases: a critical appraisal. J Am Coll Surg 2002; 195:395-408.

8. Berends FJ, Kazemier G, Bonjer HJ, Lange JB. Subcutaneous metastases after laparoscopic coloectomy. Lancet 1994; 344:58.

9. Bouvy ND, Marquet RL, Jeekel H, Bonjer HJ. Impact of gas(less) laparoscopy and laparotomy on peritoneal tumor growth and abdominal wall metastases. Ann Surg 1996; 224:694-701.

10. Curet MJ, Putrakul K, Pitcher DE, Josloff RK, Zucker KA. Laparoscopically assisted colon resection for colon carcinoma. Surg Endosc 2000; 14:1062-6. 
11. Mathew G, Watson DI, Rofe AM, et al. Wound metastases following laparoscopic and open surgery for abdominal cancer in a rat model. $\mathrm{Br}$ J Surg 1996; 83:1087-90.

12. Zmora O, Gervaz $\mathrm{P}$, Wexner SD. Trocar site recurrence in laparoscopic surgery for colorectal cancer. Surg Endosc 2001; 15:788-93.

13. Wittich P, Marquet RL, Kazemier G, Bonjer HJ. Port-site metastases after $\mathrm{CO}(2)$ laparoscopy. Is aerosolization of tumor cells a pivotal factor? Surg Endosc 2000; 14:189-92.

14. Tseng LN, Berends FJ, Wittich P, et al. Port-site metastases. Impact of local tissue trauma and gas leakage. Surg Endosc 1998; 12:1377-80.

15. Schneider C, Jung A, Reymond MA, et al. Efficacy of surgical measures in preventing port-site recurrences in a porcine model. Surg Endosc 2001; 15:121-5.

16. Balli JE, Franklin ME, Almeida JA, et al. How to prevent port-site metastases in laparoscopic colorectal surgery. Surg Endosc 2000; 14:1034-6.

17. Tai YS, Abente FC, Assalia A, Ueda K, Gagner M. Topical treatment with oxaliplatin for the prevention of port-site metastases in laparoscopic surgery for colorectal cancer. JSLS 2006; 10:160-5. 